\title{
SUCCESSFUL SURGICAL REMOVAL OFA POSTERIOR MEDIASTINAL DUMBBELL TUMOURAT VIET TIEP HOSPITAL: A CASE REPORT
}

\author{
Nguyen The May*, Do Duc Thang*, Nguyen Van Dai* Nguyen Cong Huy*, Pham Quoc Hoa*, \\ Hoang Anh Cong* Pham Thanh Tuan*, Do Manh Thang***, Le Minh Son*, Doan Quoc Hung**
}

Background: Mediastinal dumbbell tumor occurs infrequently, $10 \%$ of posterior mediastinal tumourswill develop intodumbbell tumours. Complete tumorectomy is difficult and requires multi-disciplinary approach: neurosurgery and thoracic surgery.Clinical signs arescarce, diagnosis is mainly based on chest Computerised Tomography (CT) and Magnetic Resonance Imaging (MRI) scan.

Clinical case: A43-year-old male patient was diagnosed with dumbbell tumoursonchest $\mathrm{CT}$ and MRI scan. We successfully removed the thoracic tumour by endoscopy in combination with paravertebralincision.

Key words: posterior mediastinaltumor, endoscope, dumbbell tumor,Neurogenic tumor,paravertebral

\section{BACKGROUND}

Posterior mediastinal tumours could be primary or secondary, benign or malignant, and can be found in all age groups. Approximately $10 \%$ of mediastinal tumours progresses to the spine and are called "dumb-bell" tumour. Clinical signs are scarce, the most commonly seen ones are spinal cord compression symptoms. This disease is diagnosed with chest Computerised Tomography (CT) and Magnetic Resonance Imaging (MRI) scan and the treatment of choice is surgical removal. We reported a case of a patient diagnosed with dumbbell tumour and described the surgical techniques to remove such tumours.

\section{CLINICAL CASE}

We retrospectively studied a 43-year-old male patient who was diagnosed incidentally. Chest CT and MRI scan revealed a dumbbell tumour at the level of T3-T4 with a size of $8 \times 8 \mathrm{~cm}$ and mixed density on CT scan. In addition, there are signs of enlarged intervertebral foramina and bone erosion phenomenon, enlarged intervertebral space. The drainage was removed on postoperative day 3 and the patient returned to normal life.

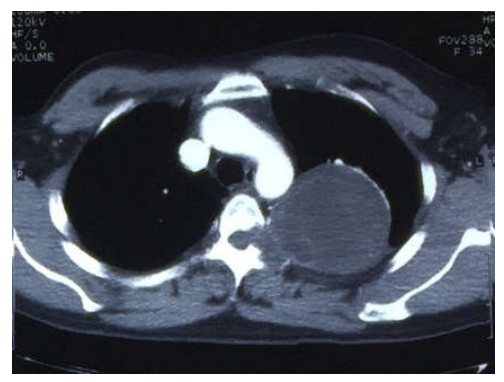

Figure 2.1 Dumbbell tumour on CT scan
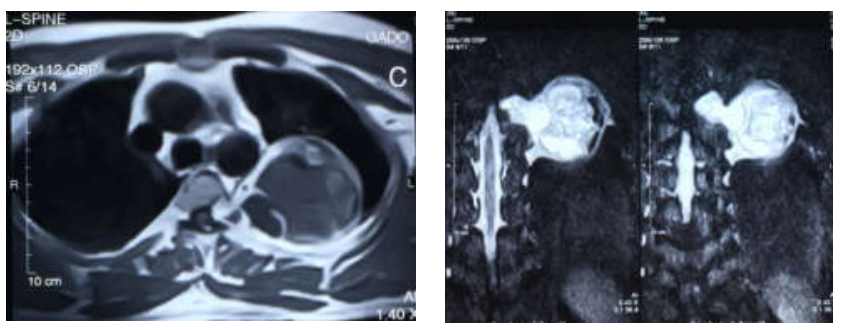

Figure 2.2 Dumbbell tumour on MRI scan

\section{SURGICAL TECHNIQUES}

\subsection{Surgical techniques.}

The patient underwent surgery under general anaesthesia with double lumen endotracheal tube.

We performed the two-staged operation in one

* Viet Tiep Hospital

** Viet Duc Hospital

Scientific director: A/ Prof. Doan Quoc Hung, PhD

Manuscript received: 01/02/2019-Accepted for publication: 23/03/2020

Scientific reviewer: A/ Prof. Dang Ngoc Hung, PhD Prof. Le Ngoc Thanh, PhD 
phase. We approached the intraspinal part of the tumour via paravertebral incision and used thoracoscopy to remove the thoracic tumour, both techniques are safe and effective.

3.1.1. First stage: Remove the spinal part of the tumour.

After general anaesthesia, the patient was positioned in prone position. We approached the tumour via paravertebral incision and hemivertebrectomy. We exposed the tumour in the spinal canal which compressed the spinal cord and therefore, was completely removed. Despite the considerable amount of blood loss $(500 \mathrm{ml})$, the circulation volume was ensured by fluid resuscitation. After surgery, a drainage was put at the paraspinal incision site.

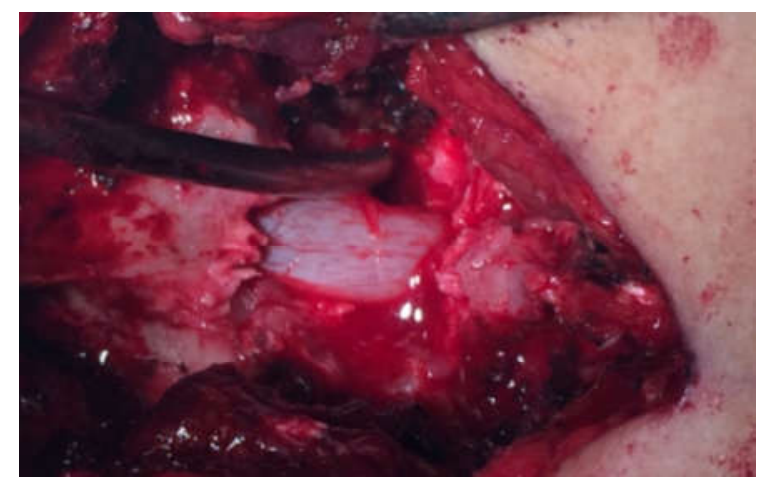

Figure 2.3 Removal of intraspinal part of the tumour

\subsubsection{Second stage:Remove thoracic part of the} tumour.

After removing the intraspinal part of the tumour, the patient was turned into his side at a $90^{\circ}$ and one lung was deflated. With this position, the deflated lung would lean forwards and the posterior mediastinal tumour would be exposed. In general, the resection of mediastinal tumours was performed with 3 trocars including one for endoscopic camera at the $6^{\text {th }}-7^{\text {th }}$ intercostal space in the anterior axillary line, one working trocar and one for suctioning. All working trocars were placed opposite to the endoscopic trocar with a reasonable distance. As the tumour had an oval shape, smooth surface and a mixed composition, we dissected the pulmonary pleura around the tumour and clipped the supplying neurons and vessels. The tumour was removed completely in an endoscopic bag before being taken out of the thoracic cavity via the $10-\mathrm{mm}$ trocar hole. After careful hemostasis, two chest drainages were put before closing the surgical incisions.

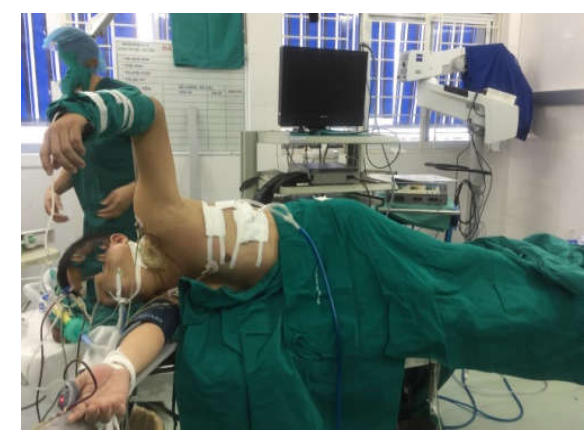

Figure 2.4 Patient position

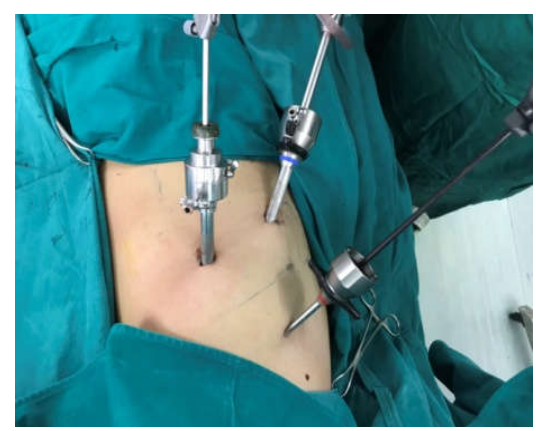

Figure 2.5 Surgical incisions

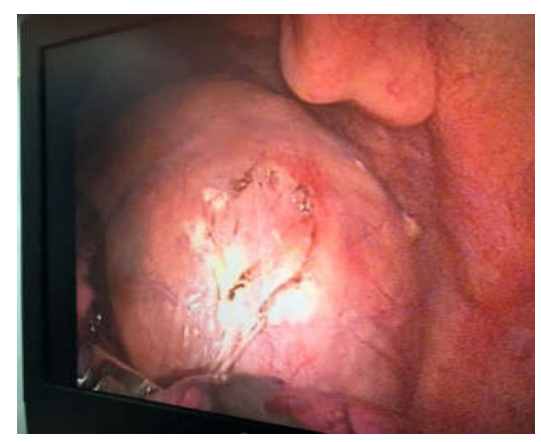

Figure 2.6 Dumbbell tumour 


\subsubsection{Results}

Both the intraspinal and thoracic components were removed completely. Chest drainages were removed on postoperative day 3. Pathology results showed that it was a benign Schwannoma.

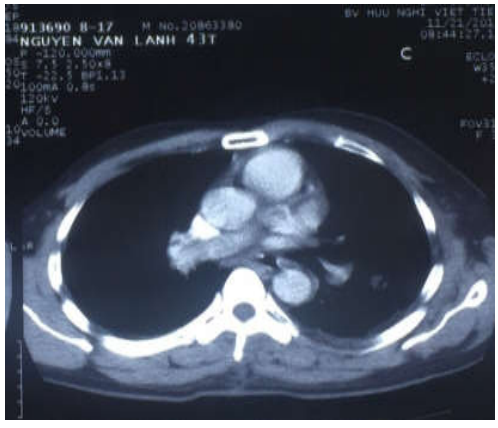

Figure 2.7 Postoperative CT scan
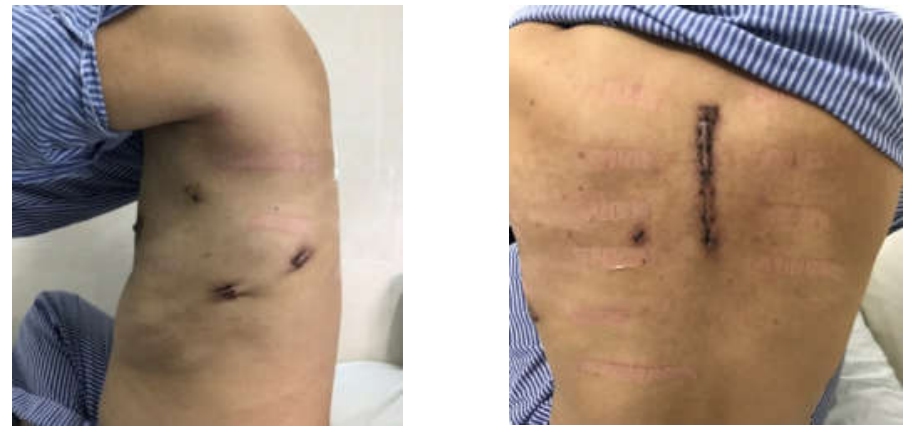

Figure 2.8 Postoperative skin incisions

\section{DISCUSSIONS}

According to many published studies, dumbbell tumours are usually benign and most of removal surgery could be beformed in a "single-stage" approach. However, this method could only be applied for thoracic tumours with sizes $<3 \mathrm{~cm}$. For tumours sizing from $3-6 \mathrm{~cm}$, the use of paravertebral incision together with rib resection in combination with thoracoscopy could reduce pain for the patient and is associated with better postoperative outcomes. For tumours sizing $>6$ $\mathrm{cm}$ or could not be removed via paravertebral incision together with rib resection, the incision should be extended posteriorly.

Nevertheless, removal of dumbbell tumours is a challenge as it requires the surgeon to approach 3 areas: spine, intervertebral foramen, and mediastinum. Although several surgical methods such as single or multiple groups combined to remove the tumour have been proposed, no definite technique has been identified. Kaiser et al concluded that most tumours could be resected with one skin incision but in some cases, a second stage or change in position may be necessary due to possible prolonged neurointervention or because the tumour is so big that it cannot be removed via paravertebral incision. Other authors including Ignacio J. Barrenechea (2006), Kyoung Hyup Nam (2017) also often change the patient position to perform two-stage and one-phase surgery. However, Yawei Li et al (2018) noted that single-stage surgery with one incision is associated with better outcomes compared with two-stage surgery due to the less invasiveness and less complications. Nevertheless, the authors also recommended the use of two-stage surgery for big, malignant tumours or those with unclear margins.

While there are not many changes in the removal of intraspinal part of tumour via paravertebral incision, techniques for resecting the thoracic part of the tumour could be applied for both open and endoscopic surgery. Together with technological advancements, the use of open chest surgery with large incision to remove mediastinal tumours is infrequent. According to Lacreuse (2007), with a wide posterior incision and muscle rection to expand the intercostal space, the patients usually complain about neuralgia or limited shoulder movements. Accordingly, thoracoscopy is increasingly implemented for posterior mediastinal surgery. However, there are many opinions regarding thorascopy for mediastinal tumours: according to the "pyramid structure" rule of Lancreneau and Hoyos, "targeted triangle" of Sasaki. However, these methods are indicated for specific locations and sizes of the tumour with the surgeon required to stay in front of the 
patient. These methods sometimes cause difficulty with the diversity of the tumour and the habits of the surgeon. Recently, Alan Sihoe (ATEP) suggested that minimal invasive surgery, video-assisted thoracoscopic surgery (VATS) are all endoscopic surgery and include:

- Three incisions: two incisions sizing $0.3-2 \mathrm{~cm}$, one $0.5-8 \mathrm{~cm}$ incision.

- All surgical manipulations are performed under video guidance (not through the incision)

- Rib retractors are prohibited

Therefore, according to Alan Sihoe's opinions, all tumours bigger than $6 \mathrm{~cm}$ could still be resected via a small incision with camera guidance. Accordingly, we performed surgical removal of the thoracic component of the tumour with a small skin incision of $2 \mathrm{~cm}$ and two trocars to remove it completely.

For tumours smaller than $6 \mathrm{~cm}$, the removal of thoracic component is usually easy but for large tumours, the removal is much harder. However, we were able to remove the tumour completely and take it out the chest cavity by cutting it into small pieces in the endoscopic bag via the small skin incision. Celalettin I Kocaturk (2017) showed that with tumours that could not be completely removed, ablation may be needed to prevent recurrence.

\section{CONCLUSIONS}

- Dumbbell tumours are relatively rare and is a special type of posterior mediastinal tumours, most of which are benign with little to no clinical manifestations and the most commonly seen symptom is signs of spinal cord compression.

- Diagnosis is based on chest CT and MRI scan.

- The treatment of choice is surgery, which is challenging and usually requires the combined approach of spinal and thoracic surgery.
- The surgery provides good outcomes, frees the spinal cord compression, short recovery time.

\section{REFERENCES}

1. Do Duc Thang, Nguyen Huu Uoc (2018). Evaluate treatment outcomes of posterior mediastinal tumours at Viet Duc Hospital from 2012 - 2017. Practical Medicine.

2. Celalettin I Kocaturk, Celal B Sezen et al (2017). Surgical approach to posterior mediastinal lesions and long-term outcomes. Asian Cardiovasc Thorac Ann, 25 (4), 287-291

3. Hong-tao Rong, MD, Yue-shan Fan (2018). Surgical technique: Management of Dumbbell and Paraspinal Tumours of the Thoracic Spine Using a Single-stage Posterolateral Approach: Case Series. Orthop Surg. 10 (4). 343-349

4. Isabelle Lacreusea, Jean Stéphane Vallab et al (2007). Thoracoscopic resection of neurogenic tumours in children. J Pediatr Surg, 42 (10), 1725-1728.

5. Kaiser, Larry. R and Thomas. L (2007). Mastery of Cardiothoracic Surgery. Williams \& Wilkins, 2nd Edition, 114-120.

6. Kyoung Hyup Nam.MD, Hyo Yeoung Ahn.MD et al (2017). One Stage Posterior Minimal Laminectomy and Video-Assisted Thoracoscopic Surgery (VATS) for Removal of Thoracic Dumbbell Tumour. $J$ Korean NeurosurgSoc, 60 (2), 257-261

7. Yawei Li, Bing Wang, Lei Li et al (2018). Posterior surgery versus combined laminectomy and thoracoscopic surgery for treatment of dumbbell-type thoracic cord tumor: A long-term follow-up. ClinNeurolNeurosurg, 166, 31-35. 